\title{
DETERMINANTS OF HARMONIC MATRICES
}

\section{J. S. MACNERNEY}

This paper is concerned with extensions of a theorem by H. S. Wall (Theorem 3 of [3]): if $M$ is a $2 \times 2$ harmonic matrix and $F$ corresponds to $M$ then det $M=1$ only in case $F_{11}=-F_{22}$.

As in [3] let $H_{n}$ denote the class of $n \times n$ harmonic matrices and $\Phi_{n}$ the class of $n \times n$ matrices $F$ of complex-valued functions from the real numbers, continuous and of bounded variation on every interval, such that $F(0)=0$. In [3] Wall has shown that the Stieltjes integral equation,

$$
M(s, t)=I+\int_{。}^{t} d F(u) \cdot M(u, t),
$$

defines a one-to-one correspondence $M \sim F$ between $H_{n}$ and $\Phi_{n}$. In studying this correspondence in a more abstract setting, the present author [1] has obtained the continuous product (or "product integral") representation,

$$
M(s, t)=\Pi^{t}\{I+d F\} \text { when } M \sim F .
$$

By using this representation, we now have the following extension of Wall's theorem cited above:

ThEOREM 1. If $M$ is in $H_{n}$ and $M \sim F$ then

$$
\operatorname{det} M(s, t)=\operatorname{Exp}\left(\sum_{1}^{n}\left[F_{p p}(t)-F_{p p}(s)\right]\right) .
$$

Proof. Let $f=\sum_{1}^{n} F_{p p}$, and $g$ be the function from the ordered real number pairs $\{s, t\}$ defined by:

$$
\operatorname{det}\{I+F(t)-F(s)\}=1+f(t)-f(s)+g(s, t) .
$$

Let $J$ be a number interval, $s$ and $t$ numbers in $J$, and $b$ a positive number.

If $u$ and $v$ are real numbers then $g(u, v)$ is a sum of products of $n$ factors, of which at least two have the form $F_{i j}(v)-F_{i j}(u)$. Thus there exist a natural number $N$ and a sequence $\left\{r_{p}, h_{p}, k_{p}\right\}_{1}^{N}$ such that if $u$ and $v$ are numbers in $J$ then

Presented to the Society, November 19, 1955; received by the editors October 17, 1955, and, in revised form, December 9, 1955. 


$$
g(u, v)=\sum_{1}^{N} r_{p}(u, v)\left[h_{p}(v)-h_{p}(u)\right]\left[k_{p}(v)-k_{p}(u)\right],
$$

where each $r_{p}$ is a bounded function from $J \times J$ to the numbers, each $h_{p}$ is one of the $F_{i j}$, and each $k_{p}$ is one of the $F_{i j}$. From the uniform continuity of the $h_{p}$ on $J$ and the bounded variation of the $k_{p}$ on $J$, it follows that there exists a positive number $c$ such that if $\left\{u_{i}\right\}_{0}^{m}$ is a monotone number sequence and $u_{0}=s$ and $u_{m}=t$ and $\left|u_{i}-u_{i-1}\right|<c$ for $i=1, \cdots, m$ then $\sum_{1}^{m}\left|g\left(u_{i-1}, u_{i}\right)\right|<b$ : hence,

$$
\begin{aligned}
& \left|\prod_{1}^{m}\left\{1+f\left(u_{i}\right)-f\left(u_{i-1}\right)\right\}-\operatorname{det} \prod_{1}^{m}\left\{I+F\left(u_{i}\right)-F\left(u_{i-1}\right)\right\}\right| \\
& \leqq \sum_{1}^{m}\left|g\left(u_{i-1}, u_{i}\right)\right| \operatorname{Exp}\left(\sum_{1}^{m}\left|f\left(u_{i}\right)-f\left(u_{i-1}\right)\right|\right. \\
& \left.\quad+\sum_{1}^{m}\left|f\left(u_{i}\right)-f\left(u_{i-1}\right)+g\left(u_{i-1}, u_{i}\right)\right|\right) \\
& \leqq b \operatorname{Exp}\left(b+2 \sum_{1}^{n} \int_{0}^{t}\left|d F_{p p}\right|\right) .
\end{aligned}
$$

Formula (3) is now apparent, since $s \prod^{t}\{1+d f\}=\operatorname{Exp}(f[t]-f[s])$.

REMARK. The formula (3) is a generalization of the well-known exponential form of the Wronskian of a fundamental set of solutions for an $n$th order linear differential equation.

It seems natural to ask for a similar result in the case of quasiharmonic matrices [2]-the statement that the $n \times n$ matrix $M$ is quasi-harmonic means that $M$ is an $n \times n$ matrix of complex-valued functions from the ordered pairs $\{s, t\}$ of real numbers, which, for each $t$, are of bounded variation in $s$ on every interval and which are quasi-continuous in $t$ for each $s$, and that, for each ordered triple $\{r, s, t\}$ of real numbers, $M(r, s) \cdot M(s, t)=M(r, t)$ and

$$
\begin{aligned}
M(s, s) & =\frac{1}{2}[M(s-, s)+M(s, s-)] \\
& =\frac{1}{2}[M(s, s+)+M(s+, s)]=I .
\end{aligned}
$$

Let $Q H_{n}$ denote the class of $n \times n$ quasi-harmonic matrices and $Q \Phi_{n}$ the class of $n \times n$ matrices $F$ of complex-valued functions from the real numbers, of bounded variation on every interval, such that

$$
[F(r)-F(r-)]^{2}=[F(r+)-F(r)]^{2}=F(0)=0 \quad \text { for each } r .
$$


In [2] we have shown that (1), with mean integrals replacing the Stieltjes integrals used by Wall, defines a one-to-one correspondence $M \sim F$ between $Q H_{n}$ and $Q \Phi_{n}$ which extends the correspondence established by Wall in [3] and which is also determined by (2).

Theorem 2. If $M$ is in $Q H_{n}$ and $M \sim F$ and $G$ is the "continuous part" of $F$ then

$$
\operatorname{det} M(s, t)=\operatorname{Exp}\left(\sum_{1}^{n}\left[G_{p p}(t)-G_{p p}(s)\right]\right) .
$$

Proof. By the "continuous part" of $F$ we mean (as in proof of Theorem 2.4 of [2]) an element $G$ of $\Phi_{n}$ such that, if $r_{1}, r_{2}, \cdots$ is a simple number sequence such that if $s$ is a number at which $F$ is not continuous then there is a natural number $k$ such that $r_{k}=s$, there is a sequence $F_{1}, F_{2}, \cdots$ of elements of $Q \Phi_{n}$ such that

(i) $F_{1}=G$ and, if $j$ is a natural number and $[a, b]$ is a number interval which does not contain $r_{j}$, then $F_{j+1}(b)-F_{j+1}(a)=F_{j}(b)$ $-F_{j}(a)$ and $F_{j+1}\left(r_{j}\right)-F_{j+1}\left(r_{j}-\right)=F\left(r_{j}\right)-F\left(r_{j}-\right)$ and $F_{j+1}\left(r_{j}+\right)$ $-F_{j+1}\left(r_{j}\right)=F\left(r_{j}+\right)-F\left(r_{j}\right)$, and

(ii) $F_{k}(s) \rightarrow F(s)$ as $k \rightarrow \infty$ for each real number $s$.

Let $F_{1}, F_{2}, \cdots$ be such a sequence and $M_{k}(s, t)=\prod_{s} \prod^{t}\left\{I+d F_{k}\right\}$ for each natural number $k$.

If $A$ is an $n \times n$ matrix of complex numbers and $A^{2}=0$ then $\operatorname{det}\{I+A\}=1$. This may easily be seen as follows: let $P$ be the function from the complex numbers defined by $P(z)=\operatorname{det}\{I+z A\}$; now $P$ is a polynomial satisfying the identity $P(z) P(-z)=1$, so that $P$ has no zero; hence $P$ is constant and its only value is $P(0)$, which is 1 .

Thus we see that $\operatorname{det} M_{k}(s, t)=\operatorname{det} M_{1}(s, t)$ for $k=1,2, \cdots$. The formula (6) now follows from Theorem 2.5 of [2] and Theorem 1 of the present paper.

\section{BIBLIOGRAPHY}

1. J. S. Mac Nerney, Stieltjes integrals in linear spaces, Ann. of Math. vol. 61 (1955) pp. 354-367.

2. - Continuous products in linear spaces, Journal of the Elisha Mitchell Scientific Society vol. 71 (1955) pp. 185-200.

3. H. S. Wall, Concerning harmonic matrices, Archiv der Mathematik vol. v (1954) pp. $160-167$.

University of North Carolina 